\title{
Hybrid Active Filter for Reactive and Harmonics Compensation in a Distribution Network
}

\author{
Victor Fabián Corasaniti, Member, IEEE, Maria Beatriz Barbieri, Senior Member, IEEE, \\ Patricia Liliana Arnera, Senior Member, IEEE, and María Inés Valla, Senior Member, IEEE
}

\begin{abstract}
The problem of reactive power and harmonics in the medium voltage level of a power distribution system is considered in this paper. Reconfiguration of the power delivery network imposes new constraints in a distribution substation so that the reactive compensation should be increased. The alternative of a shunt hybrid active filter connected to the $13.8-\mathrm{kV}$ level to enhance the power quality is analyzed in this paper. This proposal uses the existing capacitor bank to build a hybrid filter in which the complementary compensation is performed by the active filter. The performance of the hybrid filter is evaluated with extensive simulations considering reactive power, harmonics, and unbalance compensation. It shows very good behavior in steady-state and transient conditions.
\end{abstract}

Index Terms-Active filters, harmonic distortion, power distribution, power quality, reactive power.

\section{INTRODUCTION}

$\mathbf{T}$ HE INCREASE of nonlinear loads and equipment, as well as the distributed generation units in the power systems, has been demanding the compensation of disturbances caused by them [1], [2]. Voltage distortion, due to current harmonics, has become a major problem for the utilities at distribution levels. Utilities frequently encounter harmonic-related problems, such as higher transformer and line losses, reactive power and resonance problems, derating of distribution equipment, harmonic interactions between the utility and loads, reduced system stability, and reduced safe operating margins [3].

The use of traditional compensation with capacitor banks and passive filters (PFs) gives rise to harmonic propagation, i.e., harmonic voltage amplification due to resonances between line inductances and shunt capacitors. Therefore, active solutions have been continuously analyzed in the last few years [4]-[9].

One of the most popular topologies employed in harmonic compensation is the shunt active power filter (SAPF) [4], [5],

Manuscript received February 14, 2008; revised September 24, 2008. First published October 31, 2008; current version published February 27, 2009. This work was supported in part by the Instituto de Investigaciones Tecnológicas para Redes y Equipos Eléctricos, Facultad de Ingeniería, Universidad Nacional de La Plata (UNLP), in part by the FONCyT-ANPCyT, and in part by the Consejo Nacional de Investigaciones Científicas y Técnicas (CONICET).

V. F. Corasaniti, M. B. Barbieri, and P. L. Arnera are with the Instituto de Investigaciones Tecnológicas para Redes y Equipos Eléctricos-Laboratorios de Alta Tensión, Facultad de Ingeniería, Universidad Nacional de La Plata, 1900 La Plata, Argentina (e-mail: iitree@ iitree-unlp.org.ar).

M. I. Valla is with Consejo Nacional de Investigaciones Científicas y Técnicas, 1033 Buenos Aires, Argentina, and also with the Laboratorio de Electrónica Industrial, Control e Instrumentación, Área Departamental de Electrotecnia, Facultad de Ingeniería Universidad Nacional de La Plata, 1900 La Plata, Argentina (e-mail: m.i.valla@ieee.org).

Digital Object Identifier 10.1109/TIE.2008.2007997
[9]. It basically functions as a harmonic current generator feeding the needed harmonics and/or reactive currents at a certain point of the network. Several control strategies have been proposed for the SAPF [6]-[9], and those based on the generalized theory of the instantaneous active and reactive power have been the most popular [10], [11].

The particular problem of a power distribution network is considered in this paper. Reconfiguration of the network imposes new constraints on different distribution substations (DSs). Harmonics studies were performed considering the future configuration of the network. Voltage distortions in different points of the network and the working conditions of the capacitor banks were verified by means of harmonic flows [12]. A first proposal suggested increasing the existing passive compensation, but this solution introduced resonances near the fifth and seventh harmonics resulting in unacceptable distortion levels. Therefore, the alternative of an active compensation was first presented in [13]. A more accurate study of the distribution system modifies the power levels to be compensated and shows that no zero-sequence component was present and that there was very little problem of negative-sequence fundamental currents. The $132-\mathrm{kV}$ voltage system feeding the substation presents almost no harmonics or unbalances. Therefore, it appears as an ideal case to be compensated with shunt active or hybrid filters. A hybrid active filter solution is proposed in this paper. Then, it is extensively evaluated with simulations in steady state as well as transient conditions.

\section{NeTwORK DESCRIPTION}

The 132-kV network, where the DS under study is connected, works meshed and connected to a 500-kV high-voltage transmission system. In the future, the requirement to enhance the voltage profile at $13.8-\mathrm{kV}$ level demands for new compensation [12]. The loads of the different substations are mainly commercial and residential, so it is more difficult to identify the harmonic sources than in the case of industrial plants. Then, it is necessary to build a model based on the field measurements.

Fig. 1 shows the one line diagram of the system model adopted for the DS under test. A digital three-phase model of the network is constructed using the MATLAB/Simulink Power System Blockset (PSB).

The system is represented as an ideal voltage source of $132 \mathrm{kV}$ connected to three transformers of similar characteristics, 132/34.5/13.8 kV and 15/10/15 MVA. The system is modeled by its equivalent impedance related to short circuit power at $13.8 \mathrm{kV}$, which is approximately $200 \mathrm{MVA}$. There are 